\title{
Fractional models for the migration of biological cells in complex spatial domains \\ N. Cusimano ${ }^{1} \quad$ K. Burrage B $^{2} \quad$ P. Burrage $^{3}$
}

(Received 25 October 2012; revised 2 May 2013)

\begin{abstract}
Travelling wave phenomena are observed in many biological applications. Mathematical theory of standard reaction-diffusion problems shows that simple partial differential equations exhibit travelling wave solutions with constant wavespeed and such models are used to describe, for example, waves of chemical concentrations, electrical signals, cell migration, waves of epidemics and population dynamics. However, as in the study of cell motion in complex spatial geometries, experimental data are often not consistent with constant wavespeed. Non-local spatial models are successfully used to model anomalous diffusion and spatial heterogeneity in different physical contexts. We develop a fractional model based on the Fisher-Kolmogoroff equation, analyse it for its wavespeed properties, and relate the numerical results obtained from
\end{abstract}

http://journal.austms.org.au/ojs/index.php/ANZIAMJ/article/view/6283 gives this article, (C) Austral. Mathematical Soc. 2013. Published June 1, 2013, as part of the Proceedings of the 16th Biennial Computational Techniques and Applications Conference. ISSN 1446-8735. (Print two pages per sheet of paper.) Copies of this article must not be made otherwise available on the internet; instead link directly to this URL for this article. 
our simulations to experimental data describing enteric neural crestderived cells migrating along the intact gut of mouse embryos. The model proposed essentially combines fractional and standard diffusion in different regions of the spatial domain and qualitatively reproduces the behaviour of neural crest-derived cells observed in the caecum and the hindgut of mouse embryos during in vivo experiments.

\section{Contents}

1 Introduction

2 Background on fractional models

C253

3 The fractional Fisher-Kolmogoroff equation

C256

3.1 Numerical simulations . . . . . . . . . . . . . .

$\mathrm{C} 257$

3.2 Growing domain . . . . . . . . . . . .

4 Discussion

C263

5 Conclusions and future work

C265

References

C267

\section{Introduction}

Standard reaction-diffusion models exhibiting travelling wave solutions with constant wavespeed are the classic mathematical approach to describe wave phenomena in biology [11]. In the case of cell motion, the assumption that cell populations move with constant speed is often used to obtain an estimate of the speed from experimental data [9]. Enteric neural crest cells colonize the embryonic gut and move in the caudal direction (towards the posterior/inferior 
end of the body) giving rise to the enteric nervous system. In vitro assays agree with the constant wavespeed assumption [2,12], but in vivo measurements of the invasion speed of the enteric neural crest cell population are often not so straightforward. For example, the wave front is not always clearly defined, and its position may vary substantially in different experiments.

Lack of information and poor measurements also lead to wrong interpretations of biological results. In recent in vivo experiments [13], the wavespeed was determined by measuring the distance between the most caudal cell at the beginning and end of the experiment, obviously resulting in a constant estimate of the speed. Moreover, measurements were taken only in the proximal midgut and the distal hindgut (respectively the initial and the final parts of the gut observed in these experiments) because of the impossibility of observing the cell population in the caecum (the pouch between midgut and hindgut) due to its complex geometric structure and the multiple focal planes on which the cell movement occurred. When measurements were taken with a different strategy and over several short time intervals, differences in the speed became evident.

As reported by Druckenbrod and Epstein [6], in vivo measurements of neural crest invasion in embryonic mice guts show that "the enteric neural crest cell front regularly pauses at the caecum and then displays a very different pattern of migration from that found in other more proximal regions". Indeed, enteric neural crest-derived cells initially advance together as strands of connected cells. However, after pausing in the caecum, a few cells rapidly migrate forward as isolated cells and only later do the remaining cells colonize the caecum body and reach the few advanced isolated cells to again build a complex network of strands.

Evidently, a standard reaction-diffusion model cannot capture the main characteristics of the whole invasion phenomenon. One way to overcome this limitation is to modify the diffusion tensor so that it is spatially varying, but it is very difficult to validate such a model with experimental data. Another option is to consider non-local spatial models. In this article we develop 
a fractional model in space based on the Fisher-Kolmogoroff equation and attempt to validate such a model against experimental data describing enteric neural crest-derived cells migrating along the intact gut of mouse embryos. In particular, because of the link between different patterns of migration and different regions of the gut, we consider a fractional model in space with variable order. The idea behind this approach is that we want to be able to 'switch' between fractional and standard diffusion in different regions of the spatial domain.

This work is a first attempt towards a complete model for the description of the invasion of the gut by enteric neural crest-derived cells in mouse embryos.

\section{Background on fractional models}

Recently, fractional models were used to successfully describe physical phenomena characterised by anomalous diffusion or spatial heterogeneity. For example, experimental data for some phenomena [1] present non-Gaussian probability distributions and heavy tails, and these characteristics are well modelled by substituting local operators with fractional operators in space. For these operators, the flux at a given point in space depends on the whole distribution of the probability density function. Therefore, fractional models are examples of non-local models.

Given a standard reaction-diffusion problem

$$
\frac{\partial u}{\partial t}=K \Delta u+g(u),
$$

where $\mathrm{K}$ is the diffusion tensor and $\mathrm{g}(\mathrm{u})$ is the reaction term, the corresponding non-local fractional model in space is obtained by replacing the integer derivative operator by a derivative operator with a noninteger exponent $1<\alpha \leqslant 2$ (so called fractional Laplacian):

$$
\frac{\partial u}{\partial t}=-K(-\Delta)^{\alpha / 2} u+g(u) \text {. }
$$


The matrix $A$ is the spatial discretisation of the differential operator $-\Delta$, obtained with a finite difference or finite volume approach, and $A^{\alpha / 2}$ is its fractional power. A numerical solution of the fractional model is computed using a relation obtained from the matrix transfer technique [8],

$$
\mathrm{A}^{\alpha / 2}=\mathrm{VD}^{\alpha / 2} \mathrm{~V}^{-1}
$$

where $A=V D V^{-1}$ is a diagonalisation of $A$ (that is, $D$ is the diagonal matrix of eigenvalues and $V$ is the matrix of corresponding eigenvectors). In one and perhaps two spatial dimensions, this relation allows us to explicitly compute $A^{\alpha / 2}$. An explicit implementation of (2) requires only the computation of a matrix function vector product $f(A) v$ where $f$ is a suitable function of $A^{\alpha / 2}$. On the other hand, an implicit implementation requires the solution of a linear system of equations at each time step, involving the fractional power of a matrix on the left hand side. However, iterative approaches can be used to reduce this to a set of matrix function products [5].

The matrix transfer technique allows us to deal with both homogeneous Dirichlet and homogeneous Neumann boundary conditions in a straightforward manner. However, in order to handle a variable fractional order, this strategy cannot be used directly. Here we present a modification based on the matrix transfer technique that allows us to deal with zero-flux boundary conditions and a spatially varying fractional order. It is analogous to the strategy proposed by Zhuang et al. [14] for homogeneous Dirichlet boundary conditions.

Given equation (1) in one spatial dimension, let $x \in[0, \mathrm{~L}]$ and consider a spatial discretization with mesh size $h_{x}=L / N$ and nodes $x_{i}=(i-1) h_{x}$ for $i=1,2, \ldots, N+1$. Let $u_{i}$ denote the approximation of $u$ at the $i$ th node $x$. Assuming homogeneous Neumann boundary conditions,

$$
\frac{\partial u}{\partial x}=0 \quad \text { at } \quad x=0 \text { and } x=L
$$

the Laplacian is discretized in space as

$$
-\Delta \overline{\mathrm{u}} \approx \frac{1}{\mathrm{~h}_{\mathrm{x}}^{2}} \mathrm{~A} \overline{\mathrm{u}},
$$


where $\bar{u}=\left(\mathfrak{u}_{1}, \mathfrak{u}_{2}, \ldots, \mathfrak{u}_{N+1}\right)^{\mathrm{T}}$ and the matrix $A$ is the square tridiagonal matrix of order $N+1$ with $(1,2,2, \ldots, 2,1)$ on the main diagonal and $(-1,-1, \ldots,-1)$ on both the diagonals above and below the main one. We know that the eigenvalues of the real symmetric matrix $A$ are

$$
\lambda_{j}=4 \sin ^{2}\left(\frac{\pi(j-1)}{2(N+1)}\right) \quad \text { for } \quad j=1, \ldots, N+1,
$$

and the eigenvector corresponding to $\lambda_{j}$ is $v_{j}=\left(v_{j}^{(1)}, v_{j}^{(2)}, \ldots, v_{j}^{(\mathrm{N}+1)}\right)^{\mathrm{T}}$ where

$$
v_{j}^{(i)}= \begin{cases}\frac{1}{\sqrt{N+1}} & j=1, \\ \sqrt{\frac{2}{N+1}} \cos \left(\frac{\pi(j-1)(i-1 / 2)}{N+1}\right) & j \neq 1 .\end{cases}
$$

By defining $P_{j}=v_{j}$ for $j=1, \ldots, N+1$ and $P=\left(P_{1}, P_{2}, \ldots, P_{N+1}\right)$ we obtain that $\mathrm{P}$ is an orthogonal matrix such that $\mathrm{A}=\mathrm{P} \wedge \mathrm{P}^{\top}$, where $\Lambda$ is the diagonal matrix of the eigenvalues $\lambda_{j}$. From the matrix transfer technique we obtain $A^{\alpha / 2}=P \Lambda^{\alpha / 2} P^{\top}$, where $\Lambda^{\alpha / 2}$ is the diagonal matrix $\operatorname{diag}\left(\lambda_{1}^{\alpha / 2}, \ldots, \lambda_{N+1}^{\alpha / 2}\right)$. Therefore, the nonlocal operator $(-\Delta)^{\alpha / 2}$ introduced in equation (2) is discretised in space as

$$
-(-\Delta)^{\alpha / 2} \overline{\mathrm{u}} \approx-\frac{1}{\mathrm{~h}_{\chi}^{\alpha}} A^{\alpha / 2} \overline{\mathrm{u}}
$$

where

$$
A^{\alpha / 2}=\mathrm{P} \bigwedge^{\alpha / 2} \mathrm{P}^{\top}=\sum_{j=1}^{\mathrm{N}+1} \lambda_{j}^{\alpha / 2} \mathrm{P}_{j} \mathrm{P}_{j}^{\top}=\sum_{j=1}^{\mathrm{N}+1} \lambda_{j}^{\alpha / 2} v_{j} v_{j}^{\top} .
$$

Approximation (4) becomes

$$
-(-\Delta)^{\alpha / 2} \bar{u} \approx-\frac{1}{h_{\chi}^{\alpha}} \sum_{j=1}^{N+1} \lambda_{j}^{\alpha / 2}\left(v_{j}^{\top} \bar{u}\right) v_{j},
$$


which means that for $i=1, \ldots, N+1$,

$$
-(-\Delta)^{\alpha / 2} u_{i} \approx-\frac{1}{h_{x}^{\alpha}} \sum_{j=1}^{N+1} \lambda_{j}^{\alpha / 2} v_{j}^{(i)} \sum_{l=1}^{N+1} v_{j}^{(l)} u_{l} .
$$

Finally, by setting $c_{i l}=\sum_{j=1}^{N+1} \lambda_{j}^{\alpha / 2} v_{j}^{(i)} v_{j}^{(l)}$, approximation (5) is rewritten in the more concise form

$$
-(-\Delta)^{\alpha / 2} u_{i} \approx-\frac{1}{h_{x}^{\alpha}} \sum_{l=1}^{N+1} c_{i l} u_{l} .
$$

This formula is adaptable to the case of a variable fractional order $\alpha=\alpha(x)$. Let $\alpha_{i}=\alpha\left(x_{i}\right)$, then at each point of the spatial grid we only need to consider the value $\alpha_{i}$ instead of a uniform $\alpha$ for all $i=1, \ldots, N+1$.

\section{The fractional Fisher-Kolmogoroff equation}

The dimensionless variable order fractional Fisher-Kolmogoroff equation is

$$
\frac{\partial u}{\partial t}=-(-\Delta)^{\alpha(x) / 2} u+u(1-u) .
$$

By using the modification of equation (6) for the case of a variable fractional order in space, we rewrite the partial differential equation (PDE) (7) as a system of ordinary differential equations (ODEs) where for $i=1, \ldots, N+1$

$$
\frac{d u_{i}}{d t}=-\frac{1}{h_{x}^{\alpha_{i}}} \sum_{l=1}^{N+1} c_{i l} u_{l}+u_{i}\left(1-u_{i}\right), \quad c_{i l}=\sum_{j=1}^{N+1} \lambda_{j}^{\alpha_{i} / 2} v_{j}^{(i)} v_{j}^{(l)}
$$

In matrix form we therefore obtain

$$
\overline{\mathrm{u}}^{\prime}=\mathrm{B} \overline{\mathrm{u}}+\overline{\mathrm{g}},
$$


where $\bar{g}=\left(g_{1}, \ldots, g_{N+1}\right)^{\top}$ with $g_{i}=u_{i}\left(1-u_{i}\right)$ for all $i$ and the components of matrix B are

$$
\mathrm{B}_{i l}=-\frac{1}{\mathrm{~h}_{x}^{\alpha_{i}}} \sum_{j=1}^{\mathrm{N}+1} \lambda_{j}^{\alpha_{i} / 2} v_{j}^{(i)} v_{j}^{(l)} \text {. }
$$

To obtain a numerical approximation of the solution of equation (9) we use the Matlab ODE solver ode15s. The particular form of the nonlinear system (9) allows us to provide explicitly the Jacobian matrix of the right hand side and sensibly speed up the computation of the solution. Indeed, the Jacobian is simply $\mathrm{J}=\mathrm{B}+\mathrm{G}$ where $\mathrm{G}$ is the diagonal matrix with entries $1-2 u_{i}$ for $i=1,2, \ldots, N+1$.

\subsection{Numerical simulations}

Let $x \in[0,250]$ and consider a uniform spatial grid with mesh size $h_{x}=0.125$. Given the initial condition

$$
u_{0}(x)= \begin{cases}1 & 0 \leqslant x<10 \\ e^{-10(x-10)} & 10 \leqslant x \leqslant 250\end{cases}
$$

and assuming homogeneous Neumann boundary conditions, the evolution in time (for $t \in[0,100]$ ) of the numerical solution of equation (7) with fractional order $\alpha(x)=1.96$ for all $x$ is shown in Figure 1. We compute the solution at regular time intervals of $\Delta t=0.02$ but the solution profile is plotted every 125 timesteps.

Unlike the case of standard diffusion, there are no travelling wave solutions moving with constant speed. Indeed, in agreement with analytical results provided by Engler [7], we observe a rapid deformation of the solution profile towards the stable steady state $\mathfrak{u}=1$ of the spatially homogeneous version of the PDE (7). 


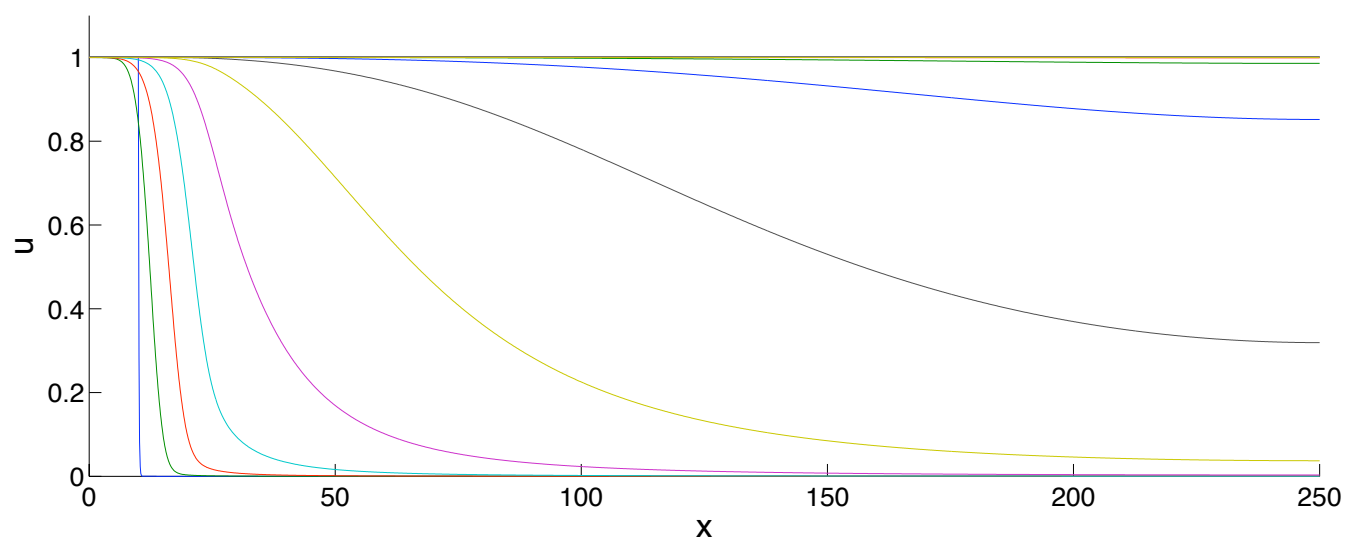

Figure 1: Evolution of the numerical solution for $\alpha=1.96$. Solution profile plotted at regular time intervals of $\Delta t=2.5$.

We now use the variable fractional order defined as

$$
\alpha(x)= \begin{cases}1.96 & x \leqslant 125 \\ (1.96-2)(x-126)(125-126)^{-1}+2 & 125<x<126 \\ 2 & x \geqslant 126\end{cases}
$$

which corresponds to considering the fractional modification in the subinterval $[0,125]$ and the standard Fisher-Kolmogoroff equation in $[126,250]$ with linear interpolation between the two levels of $\alpha$. The evolution in time of the solution profile is shown in Figure 2.

The rapid movement of the solution profile towards the stable steady state slows down as the solution moves closer to the second half of the spatial interval where the dynamics is governed by the standard Fisher-Kolmogoroff equation. Indeed, in this subinterval, the fractional diffusion is not effective anymore and the solution evolves to a travelling wave moving with constant speed.

It is possible to characterise such solutions in terms of their level sets. For a fixed value $w \in(0,1)$, the corresponding level set is defined as the set 


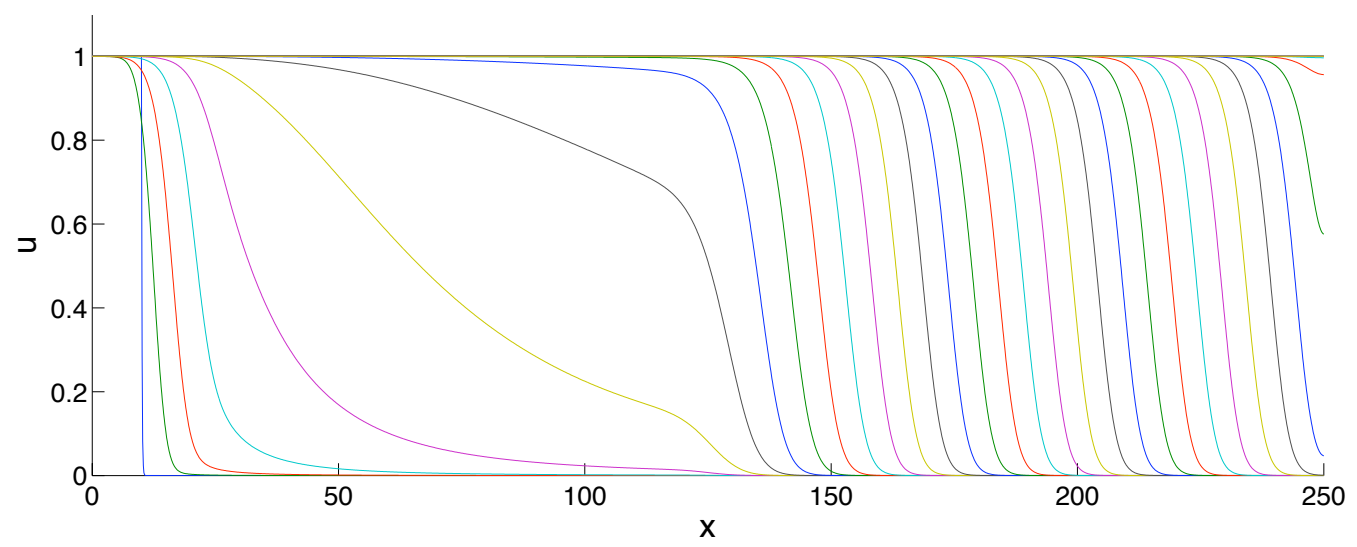

Figure 2: Evolution of the numerical solution for $\alpha=\alpha(x)$ given by equation (12). Solution profile plotted at regular time intervals of $\Delta t=2.5$.

$\left\{\left(x_{w}(t), t\right) \mid u\left(x_{w}(t), t\right)=w\right\}$. In the simulation with variable fractional order, we track the position $x_{w}\left(t_{k}\right)$ for different values of $w$ at each timestep $t_{k}$ and plot these positions as functions of time to obtain Figure 3. Note that $x_{w}(t)$ is quickly affected by the fractional power $\alpha(x)<2$, resulting in a rapid advancement of the level set positions. However, once the solution profile approaches the second half of the spatial interval (where $\alpha=2$ ), the advancment of the level sets slows down and continues linearly in time.

By numerically approximating the migration speed $c_{w}(t)$ at each time step $t_{k}$ with the incremental ratio

$$
c_{w}\left(t_{k}\right)=\frac{x_{w}\left(t_{k}+\Delta t\right)-x_{w}\left(t_{k}\right)}{\Delta t}
$$

we see from Figure 4 that, as expected, we have a quick acceleration, followed by a deceleration and an adjustment of the speed around a constant value. This constant value is exactly the minimum wavespeed for the standard Fisher-Kolmogoroff equation, that is, $c_{\min }=2$. The smaller the value of $w$, the sooner the effect of the fractional and standard diffusion on the evolution of $x_{w}(t)$ (and consequently on $c_{w}(t)$ ) becomes evident. 


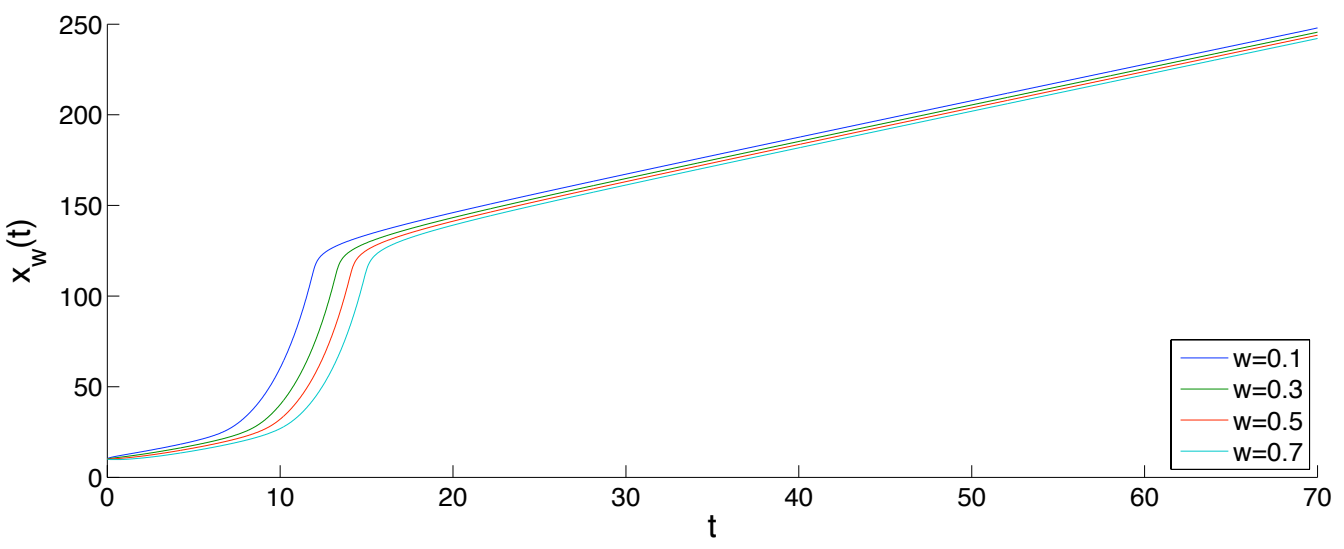

Figure 3: Position of different level sets in time.

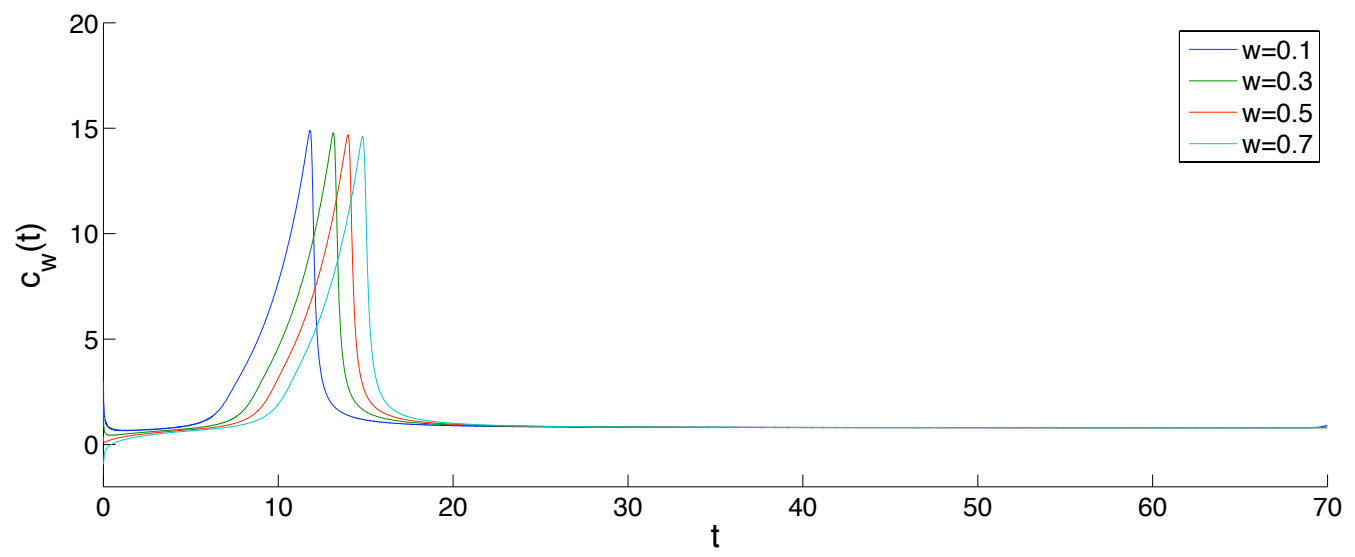

Figure 4: Speed as a function of time. 


\subsection{Growing domain}

So far we have assumed that the underlaying domain does not change throughout the simulation. Here we follow a similar analysis of the Fisher-Kolmogoroff equation, but now assume a uniformly growing domain. Let us consider (for the sake of clarity) the general dimensional form of the Fisher-Kolmogoroff equation in one spatial dimension,

$$
\frac{\partial u}{\partial t}=D \frac{\partial^{2} u}{\partial x^{2}}+k u(1-u),
$$

where $\mathrm{D}$ and $\mathrm{k}$ are positive parameters. As described by Binder et al. [3], domain growth "implies that there is a local velocity $v(x, t)$ such that a point $x$ moves to the point $x+v(x, t) \Delta t$ during a small time interval $\Delta t$ ". The uniform growth of the domain contributes an additional convective term to the flux and therefore the Fisher-Kolmogoroff equation in this case is

$$
\frac{\partial u}{\partial t}=D \frac{\partial^{2} u}{\partial x^{2}}+k u(1-u)-\frac{\partial}{\partial x}(v u)
$$

for $t>0$ and $0<x<L(t)$. In our simulations we assume that the one dimensional domain elongates in time as a logistic function,

$$
\mathrm{L}(\mathrm{t})=\frac{\mathrm{L}_{\infty}}{1+\left(\mathrm{L}_{\infty}-1\right) e^{-\mathrm{ct}}},
$$

where $\mathrm{L}_{\infty}$ and $\mathrm{c}$ are two parameters specific to a given problem.

By introducing a new spatial variable $z=x / L(t)$, we rewrite the model on a spatial interval with fixed length and equation (14) becomes

$$
\frac{\partial u}{\partial t}=\frac{D}{L(t)^{2}} \frac{\partial^{2} u}{\partial z^{2}}+k u(1-u)-\frac{1}{L(t)} \frac{d L}{d t} u
$$

for $t>0$ and $0<z<1$. The corresponding fractional modification of equation (16) is obtained simply by replacing the second order derivative 


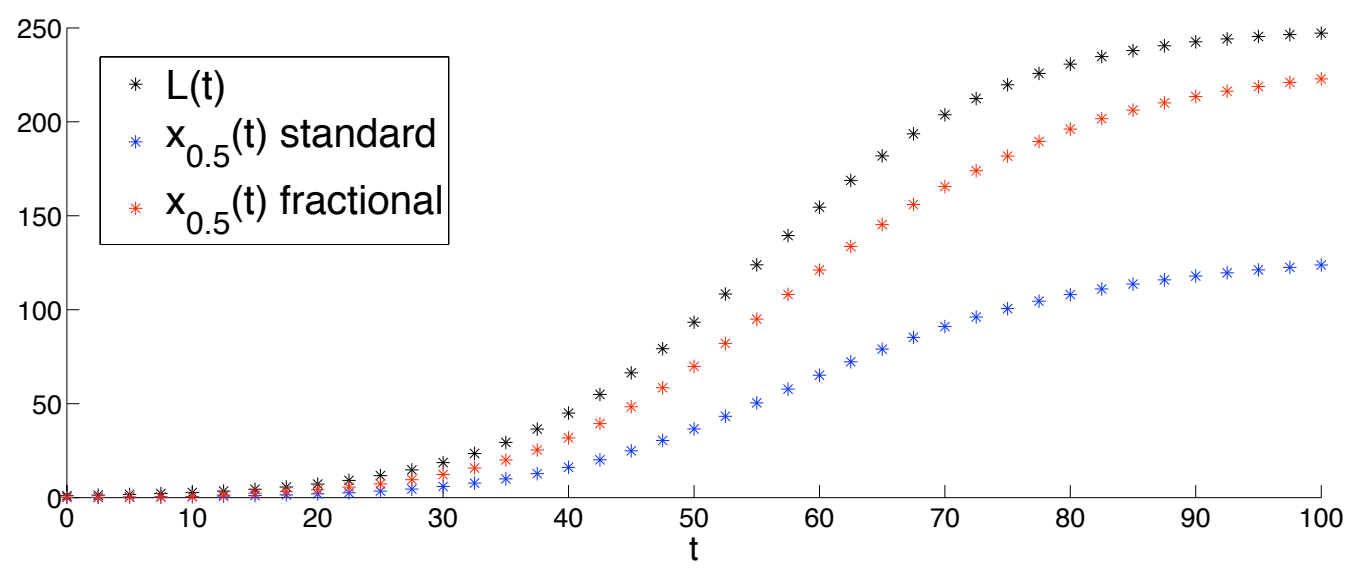

Figure 5: Domain length and front position as functions of time.

in space with the fractional operator. Here we compare the solution of the standard diffusion case with the one obtained with a spatially variable $\alpha(z)$, defined as $\alpha(z)=1.96$ in the first half of $[0,1]$ and $\alpha(z)=2$ in the second half with linear interpolation between the two levels. For both cases we use the numerical strategy previously described to discretize the PDE in space and then compute the evolution in time of the solution on the fixed domain, that is, for $z \in[0,1]$. By using the definition of $z$, we obtain the results in terms of the original variable $x$. In the fractional case the solution is computed by exploiting prior knowledge of the analytical form of the eigenvalues and eigenvectors of the matrix of the spatial discretization.

Exploiting the concept of level sets introduced previously, we track the location of the front and relate it to the temporally increasing length of the domain. Figure 5 shows the growing length of the spatial interval (black) and the advancing position $x_{0.5}(t)$ as functions of time, for both the standard diffusion case (blue) and its fractional modification (red).

We observe that the advancing front in the standard diffusion case is no longer linear and is affected by the way the underlying domain elongates. However, there is still a big difference in the front location of the standard and fractional 


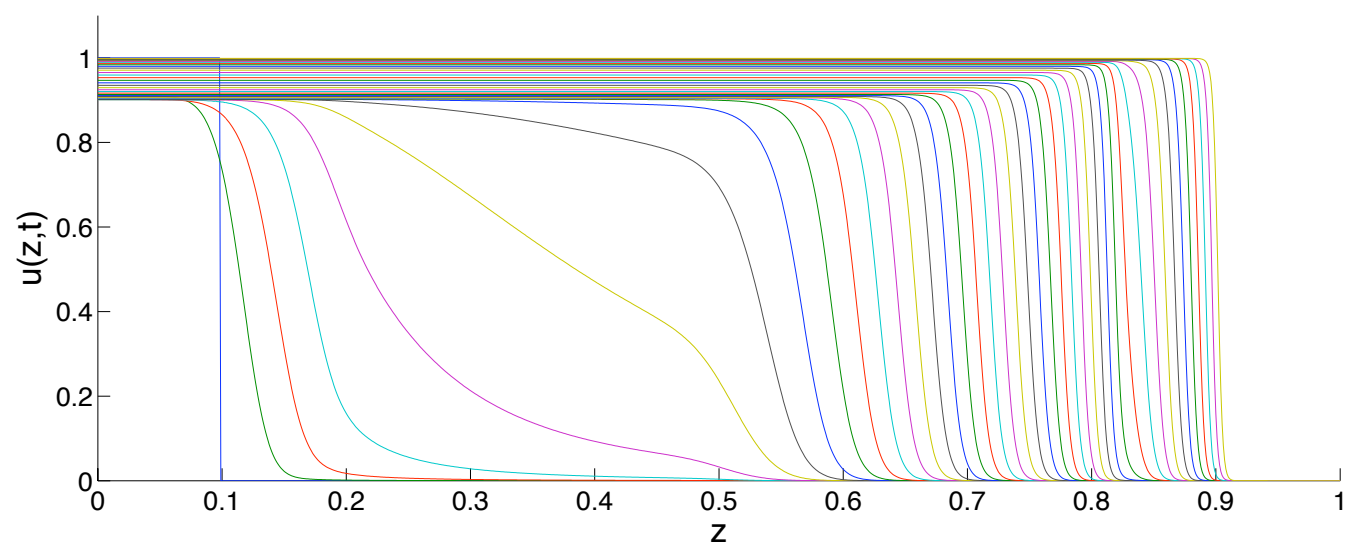

Figure 6: Solution profile on the fixed spatial interval $[0,1]$ (fractional modification). Solution profile plotted at regular time intervals of $\Delta t=2.5$.

models. For the fractional modification (shown in Figure 6) in terms of the variable $z$, that is, on the fixed interval $[0,1]$, the front accelerates in the region where $\alpha(x)<2$, and this acceleration allows the front to travel faster across the growing spatial domain than in the corresponding model with standard diffusion.

\section{Discussion}

Biological data provided by Druckenbrod and Epstein [6] on the pattern and the average speed of invasion of the gut in mouse embryos is summarized as follows. Enteric neural crest-derived cells colonize the gut predominantly in the form of strands of connected cells. At E10.5 (embryonic stage of development) the front is in the ileum and advances caudally at an average speed of $45 \mu \mathrm{m} \mathrm{h}^{-1}\left( \pm 10.8 \mathrm{SE}^{1}\right)$ but slows down to $32 \mu \mathrm{m} \mathrm{h}^{-1}$ at E11.25 when approaching the nascent caecum. At E11.5, for a period of 8-12 hours, the

\footnotetext{
${ }^{1}$ Standard error.
} 
enteric neural crest cell population is concentrated at the caecal base and does not migrate forward. A number of single cells break from the strands, move quickly into the caecal body as isolated cells, and after approximately 12 hours begin to come together to form short strands. After the initial front, the remaining cells entre the caecal body and join with the short strands, forming a complete network again. From E11.5 to E12.5 the wavefront advances through the caudal axis of the caecal body at an average speed of $23 \mu \mathrm{m} \mathrm{h}^{-1}( \pm 6.3 \mathrm{SE})$. This value includes the period when the cells pause at the caecal base (from E11.5 to E12.0). Finally, from E12.5 to E13.25, the wavefront moves at an average speed of $30 \mu \mathrm{m} \mathrm{h}^{-1}( \pm 7.8 \mathrm{sE})$ along the caudal axis of the mid-colon. At E13.5 the enteric cells approach the termination of the bowel.

Since the average speed of the cells in the caecal body includes the time the cells spend at rest, the average speed of the moving cells must be much higher than $23 \mu \mathrm{m} \mathrm{h}^{-1}$. The pause period is in fact almost as long as the time required to colonize the entire caecum body.

The speeds obtained from these experiments show that the dynamics of the invasive process reflects particular properties of the gut structure along which the migration occurs and changes according to the location of the front in the domain. In particular, the more complex geometric structure of the caecum and the heterogeneity of the caecal tissue result in an anomalous behaviour of the cell population. We interpret the variable $u$ as the cell concentration of neural crest-derived cells along the longitudinal axis of the gut, and associate different values of $\alpha$ with different intervals of our spatial domain to reflect particular spatial connectivity patterns specifically observed in those intervals. With this interpretation we successfully relate the qualitative behaviour of our numerical results presented in Section 3.1 with the invasive process of the mouse embryonic gut after the pause at the caecum base. Indeed, the pattern of cell invasion observed in the caecum body presents the same features observed for the numerical solution in the first half of our spatial domain, where $\alpha(x)<2$. From our analysis of the position of level sets for different values of $w \in(0,1)$, we observe a very fast advancement of $x_{w}(t)$ when we consider a low value of $w$ (corresponding to a low cell concentration) followed 
by the rapid invasion by the rest of the cell population of the subinterval representing the caecum body (high concentration level corresponding to a value of $w$ close to 1 ). On the other hand, in the colon (structure located at the beginning of the hindgut) and the final part of the gut observed in the experiments, the front of the reestablished complete network of strands is simulated by a standard reaction-diffusion process.

At this stage, because of the lack of more detailed results on the front location and the speeds as functions of time, we are not able to validate our model any further but we recognize that the qualitative behaviour of the invasion process after the period of pause at the caecum base is well-captured by our approach. Moreover, even if the assumption of a growing underlying tissue seems reasonable to model mice gut during the early stages of development, the lack of measurements of the tissue length for the particular type of experiment analysed does not allow us to decide whether the model accounting for gut elongation is preferable compared to the one on a fixed domain.

\section{Conclusions and future work}

The variable order fractional model proposed in this article is a new approach in the context of cell migration. As discussed in Section 4, our model provides promising results in terms of reproducing the qualitative behaviour of mouse enteric neural crest invasion in vivo. The key aspect of this modeling approach is the possibility of changing from fractional to standard diffusion in different subintervals of the spatial domain, combining regions characterised by spatial heterogeneity with others where the invasion dynamics agrees with the constant wavespeed assumption.

The major limitation of the fractional Fisher-Kolmogoroff model with variable order proposed in this article is that we cannot reproduce the migrating behaviour observed in the midgut and at the caecum base, that is, a front initially advancing with constant speed and subsequently decelerating and 
pausing. Recent studies [4] link the pause at the caecum base with an inhibitory effect due to some ligands of receptors strongly expressed in the caecum, suggesting a chemotaxis effect which we will introduce into our model.

Studies on how a subpopulation of embryonic cells travel long distances, respond to tissue growth and reach a target present interesting insights on the role of heterogeneity in an invading cell population. McLennan et al. [10] proposed a fully integrative experimental-modelling approach, analysed the migratory behaviour of cranial neural crest cells of a living chick embryo and showed that a simple cell chemotaxis model is insufficient to explain the experimental evidence. To reproduce a successful invasion of the domain in silico the model was refined by introducing two neural crest cell populations (namely leading and trailing cells) which respond differently to local microenvironmental signals. However, unlike our model, McLennan et al. [10] considered a two dimensional domain growing in time along the $x$ axis according to a logistic function. The neural crest cell population had its own migrating ability but at the same time it was dragged along the domain by such growth. Rather than being derived from first principles, the choice of a logistic function for the domain growth was justified only by the need to fit model predictions to experimental data.

In this article we do not consider the two dimensional extension of the model but focus on the effect of a spatially varying fractional order reflecting different spatial connectivity properties of the gut structures in one dimension. We also have not dealt in detail with the effects of domain growth on the solution behaviour. However, we ran simulations taking into account both of these aspects and we report here some general observations, leaving the detailed analysis for future work.

The idea behind a two dimensional extension is to model the neural crestderived cell concentration on the gut walls, where the gut is idealised as a long thin cylinder. We identify our two dimensional domain with the external surface of this cylinder cut along the gut length ( $x$ direction) and 
therefore model the problem on a long thin rectangle. The one dimensional initial condition considered in this article is extended in a natural way to the two dimensional problem by simply defining a uniform behaviour along the $y$ direction (representing the cross-section of the gut). For a fixed rectangular domain, we observe that the behaviour of the two dimensional solution (both in the standard diffusion case and in the case of fractional diffusion with variable fractional order $\alpha=\alpha(x)$ ) preserves the invariance along the $y$ axis and therefore for all values of $y$ the solution profile along $x$ is exactly the same.

Previous studies on gut elongation and cell migration in the standard diffusion case [3] showed that domain growth impacts both cell migration and proliferation and the choice of a particular function (or combination of functions) for the uniform (nonuniform) domain growth is fundamental in determining the solution behaviour. Here we only described the general methodology to be applied in the case of uniform growing one dimensional domain and provided some considerations for a specific functional form of $L(t)$. We do not exclude the fact that the choice of a different $\mathrm{L}(\mathrm{t})$ or the assumption of nonuniform domain growth might produce qualitatively different results. Moreover, we acknowledge that a two dimensional model and the assumption of a growing domain might lead to interesting results because the change in geometry (especially if nonuniform throughout the domain) is likely to affect the solution behaviour and could be responsible for changes in the speed of the advancing neural crest cell front. In light of all these considerations, the investigation of this interaction will be the next step towards the simulation of the complete phenomenon of cell invasion in mouse embryonic gut.

\section{References}

[1] R. J. Adler, R. E. Feldman and M. S. Taqqu. A practical guide to heavy tails: Statistical techniques and applications. Birkäuser, 1998. C253 
[2] I. J. Allan and D. F. Newgreen. The origin and differentiation of enteric neurons of the intestine of the fowl embryo. The American Journal of Anatomy, 157, 137-154, 1980. doi:10.1002/aja.1001570203 C252

[3] B. J. Binder, K. A. Landman, M. J. Simpson, M. Mariani and D. F. Newgreen. Modeling proliferative tissue growth: A general approach and an avian case study. Physical Review E (Statistical, Nonlinear, and Soft Matter Physics), 78(3), 1-13, 2008. doi:10.1103/PhysRevE.78.031912 C261, C267

[4] M. A. Breau, A. Dahmani, F. Broders-Bondon, J. P. Thiery and S. Dufour. $\beta 1$ integrins are required for the invasion of the caecum and proximal hindgut by enteric neural crest cells. Development, 136, 2791-2801, 2009. doi:10.1242/dev.031419 C266

[5] K. Burrage, N. Hale and D. Kay. An efficient implementation of an implicit FEM scheme for fractional-in-space reaction-diffusion equations. SIAM Journal on Scientific Computing, 34(4), A2145-A2172. doi:10.1137/110847007 C254

[6] N. R. Druckenbrod and M. L. Epstein. The patterns of neural crest advance in the cecum and colon. Developmental Biology, 287, 125-133, 2005. doi:10.1016/j.ydbio.2005.08.040 C252, C263

[7] H. Engler. On the speed of spread for fractional reaction-diffusion equations. International Journal of Differential Equations, 2010, Article ID 315421, 2010. doi:10.1155/2010/315421 C257

[8] M. Ilić, F. Liu, I. Turner and V. Anh. Numerical approximation of a fractional-in-space diffusion equation (II)-with nonhomogeneous boundary conditions. Fractional Calculus and Applied Analysis, 9, 333-349, 2006. http://eprints.qut.edu.au/23835/ C254

[9] P. K. Maini, D. L. S. McElwain and D. Leavesley. Travelling waves in a wound healing assay. Applied Mathematics Letters, 17, 575-580, 2004. doi:10.1016/S0893-9659(04)90128-0 C251 
[10] R. McLennan, L. Dyson, K. W. Prather, J. A. Morrison, R. E. Baker, P. K. Maini and P. M. Kulesa. Multiscale mechanisms of cell migration during development: theory and experiment. Development, 139, 2935-2944, 2012. doi:10.1242/dev.081471 C266

[11] J. D. Murray. Mathematical Biology I and II. Springer Verlag, 2003. $\mathrm{C} 251$

[12] M. J. Simpson, D. C. Zhang, M. Mariani, K. A. Landman and D. F. Newgreen. Cell proliferation drives neural crest cell invasion of the intestine. Developmental Biology, 302, 553-568, 2007. doi:10.1016/j.ydbio.2006.10.017 C252

[13] H. M. Young, A. J. Bergner, R. B. Anderson, H. Enomoto, J. Milbrandt, D. F. Newgreen and P. M. Whitington. Dynamics of neural crest-derived cell migration in the embryonic mouse gut. Developmental Biology, 270, 455-473, 2004. doi:10.1016/j.ydbio.2004.03.015 C252

[14] P. Zhuang, F. Liu, V. Anh and I. Turner. Numerical methods for the variable-order fractional advection-diffusion equation with a nonlinear source term. SIAM Journal on Numerical Analysis, 47(3), 1760-1781, 2009. doi:10.1137/080730597 C254

\section{Author addresses}

1. N. Cusimano, School of Mathematical Sciences, Queensland University of Technology, Brisbane, QLD 4001, Australia. mailto:nicole.cusimano@student.qut.edu.au

2. K. Burrage, Oxford University Computing Laboratory, Wolfson Building, Parks Road, Oxford OX1 3QD, UK. mailto:kevin. burrage@cs.ox.ac.uk

3. P. Burrage, School of Mathematical Sciences, Queensland University of Technology, Brisbane, QLD 4001, Australia. 
mailto:pamela. burrage@qut.edu.au 